\title{
Male infertility due to globozoospermia
}

INSERM

\section{Source}

INSERM. (1999). Orphanet: an online rare disease and orphan drug data base. Male infertility due to globozoospermia. ORPHA:171709

Male infertility due to globozoospermia is a male infertility due to sperm disorder characterized by the presence, in sperm, of a large majority of round-headed spermatozoa that lack the acrosome and have an aberrant nuclear membrane and midpiece defects. The acrosomeless spermatozoa is not able to penetrate the zona pellucida and thus fertilization failures, even with intracytoplasmic spem injection, are frequent. 